\title{
Identification of neural structures involved in stuttering using vibrotactile feedback
}

Oli Cheadle, Clarissa Sorger, Peter Howell*

Division of Psychology and Language Sciences, University College London, UK

*Correspondence to: Peter Howell, Department of Experimental Psychology, University College London, Gower Street, London WC1E 6BT,

United Kingdom. E-mail: p.howell@ucl.ac.uk (Peter Howell)

Running title: Vibrotactile feedback and stuttering

Conflict of Interest: None 


\begin{abstract}
Feedback delivered over auditory and vibratory afferent pathways has different effects on he fluency of people who stutter (PWS). These features were exploited to investigate the neural structures involved in stuttering. The speech signal vibrated locations on the body (vibrotactile feedback, VTF). Eleven PWS read passages under VTF and control (no-VTF) conditions. All combinations of vibration amplitude, synchronous or delayed VTF and vibrator position (hand, sternum or forehead) were presented. Control conditions were performed at the beginning, middle and end of test sessions. Stuttering rate, but not speaking rate, differed between the control and VTF conditions. Notably, speaking rate did not change between when VTF was delayed versus when it was synchronous in contrast with what happens with auditory feedback. This showed that cerebellar mechanisms, which are affected when auditory feedback is delayed, were not implicated in the fluency-enhancing effects of VTF, suggesting that there is a second fluency-enhancing mechanism.
\end{abstract}

Key words: Vibrotactile feedback; stuttering; altered auditory feedback; EXPLAN. 


\section{Introduction}

Stuttering interrupts the forward flow of speech (Howell, 2010). Meta-analyses have identified three replicable and distinctive anomalous neural patterns that are associated with stuttering (Belyk, Kraft \& Brown, 2014; Brown, Ingham, Ingham, Laird \& Fox, 2005; Budde, Barron \& Fox, 2014): 1) activity in auditory cortex is reduced; there is over-activity in 2) the right frontal operculum and/or anterior insula; and 3) the cerebellar vermis. Stimulating these affected brain regions could have an impact on stuttering (Howell \& Lu, 2016). One way of doing this is to deliver exteroceptive sensory inputs that transmit to the regions where activity is anomalous and determine what effects these modulations have on the fluency of PWS. Applying this logic, auditory stimulation with metronome clicks (Howell \& El-Yaniv, 1987) or masking noises (Cherry \& Sayers, 1956) ameliorated stuttering. Presenting a changed version of the speaker's own voice (altered auditory feedback, AAF) by delaying (DAF) it or altering its spectral content (FSF) also affected the fluency of PWS (Howell, El-Yaniv \& Powell, 1987). These improvements under AAF have been replicated and extended in research (for reviews: Howell, 2004 and Kalinowski \& Saltuklaraglu, 2006) and clinical settings (for review: Lincoln, Packman, \& Onslow, 2006). A prosthetic device that delivers DAF and FSF whilst PWS speak is available (Stuart, Xia, Jiang, Jiang, Kalinowski \& Rastatter, 2003).

A related issues concerns whether stuttering changes when non-auditory stimuli are presented to PWS whilst they speak. These inputs employ transmission pathways that by-pass or transmit over additional afferent pathways to those involved in audition. These properties provide a way of determining the role that the included and excluded neural structures play in stuttering. The most extensively investigated non-auditory modality is vibration. In one procedure, the speaker's vocal output drives vibration transducers attached to the body (vibrotactile feedback, VTF). VTF and AAF are similar in that they both: 1) change speech feedback from its normal state; and 2) improve the fluency of PWS (Kuniszyk-Jozkowiak, Smolka \& Adamczyk, 1996; Snyder, Blanchet, Waddell \& Ivy, 2009; Waddell, Goggans \& Snyder, 2012). VTF and AAF are transmitted over different neural pathways, at least to levels below the thalamic nuclei (see Hendelman, 2005, and Juliano \& Mclaughlin, 1999 respectively for 
reviews of the auditory and vibratory pathways). Consequently, VTF pathways bypass the sub-thalamic afferent auditory pathways that project from the cochlea to the cerebellum and auditory cortex.

The present study exploited the known differences between auditory and vibratory afferent architectures and the changes to speech control that occur under AAF and VTF in PWS, to investigate neural mechanisms for facilitating fluency. As background: 1) the pathways to somatosensory cortex that VTF takes are described and compared with the auditory pathways; 2) the ways VTF influences the fluency of PWS are reviewed; 3) a neural model, that proposes how auditory inputs improve the fluency of PWS, is described. One way in which the model could be revised to account for how VTF affects the speech of PWS by incorporating the observations about projection pathways is considered; and 4) the predictions for the current study that derive from the revised model are presented.

\subsection{Vibratory and auditory pathways}

Two types of mechanoreceptor in the skin are responsible for detecting vibration: Meissner's corpuscles are sensitive to frequencies between 30 and $50 \mathrm{~Hz}$; Pacinian corpuscles detect vibrations between 100 and $400 \mathrm{~Hz}$ (peak sensitivity of $250 \mathrm{~Hz}$ ) (Griffin, 1990; Siegel \& Sapru, 2006).

The main sensory trigeminal cranial nerve $(\mathrm{CN})$ and posterior column-medial lemniscal (non-CN) pathways transmit vibrotactile information to primary somatosensory cortex. The trigeminal $\mathrm{CN}$ enters the brainstem at the level of the mid-pons and ascends via the trigeminal lemniscus, to the thalamus, and from there to primary somatosensory cortex (Juliano \& Mclaughlin, 1999). This pathway carries information about discriminative touch for the face and top of the head. The fasciculus gracilis, which forms part of the posterior column-medial lemniscal pathway, provides information about vibration from the upper part of the body ( $\mathrm{t} 6$ and above, including the arms) to the primary somatosensory cortex via the medulla and thalamus. Neither vibratory pathway transmits via the cerebellum. 
The afferent auditory system projects from the cochlear nucleus to the inferior colliculus and then via the medial geniculate nucleus to auditory cortex (Hendelman, 2005). The medial geniculate nucleus is part of the thalamic relay system and auditory projections after this may meet up with the post-thalamic vibration pathways. Auditory stimulation activates cerebellar pathways: There are direct connections from the cochlear nucleus to the vermis area of the cerebellum (part of its medial division) in mammals (Huang, Liu \& Huang, 1982; Niemer \& Cheng, 1949; Zhang, Sun \& Jen, 1990). The anatomy of all parts of the cerebellum is similar (Edge, Marple-Horvat \& Apps, 2003), allowing the inputs to medial cerebellum to activate the lateral cerebellum directly.

A MEG study by Caetano and Jousmaki (2006) showed that $200 \mathrm{~Hz}$ vibrotactile stimulation activated primary somatosensory as well as auditory cortex and activation of the latter was not due to sound artefacts. Consequently, vibration and audition may share a pathway which, anatomical considerations suggest, would have to lie above the thalamus (Hendelman, 2005; Juliano \& Mclaughlin, 1999). The post-thalamic structures could be responsible for the fluency-enhancing effects of VTF and any form of AAF that is transmitted to them. In contrast, any cerebellar mechanisms involved in fluency-enhancement could only be accessed by auditory inputs because they transmit to this structure whereas vibrotactile stimulation by-passes them.

\subsection{Effects of VTF on PWS}

Kuniszyk-Jozkowiak et al. (1996) investigated how synchronous and asynchronous auditory, vibratory and visual speech feedback affected fluency (percentage syllables stuttered, \%SS) and speaking rate (number of syllables uttered per second). Synchronous (0 ms delay) and asynchronous (delays of 50,100 and $160 \mathrm{~ms}$ ) feedback were investigated in all modalities. \%SS and speaking rate reduced significantly as feedback delay increased for all modalities. Both measures reduced more under Delayed Auditory Feedback (DAF) than under delayed VTF. 
PWS in Snyder et al.'s (2009) study held their thyroid cartilage between thumb and index finger. This synchronous VTF reduced stuttering frequency by $72 \%$ when compared to a condition where there was no VTF. Speaking rate was not examined.

Waddell et al. (2012) picked up vibration on the throat either by microphone or by accelerometer and used this to deliver synchronous VTF to tactile stimulators held between the index finger and thumb. Stuttering frequency reduced significantly and depended on whether a microphone or an accelerometer was used to pick up the vibration; accelerometer-driven feedback led to most improvement.

\subsection{Model for processing auditory inputs that are asynchronous with vocalization and modifications for incorporating VTF effects}

Auditory and motor inputs are synchronous when speech is fluent. For example, an efferent copy of fluent speech plans has the same time pattern as the corresponding speech output (Howell \& Sackin, 2002). Differencing the timing patterns of these synchronous inputs would null them and absence of activity could signal that speech is fluent and no change to speech control is necessary (Howell, 2010).

DAF creates a situation where the sound of the speaker's voice is asynchronous with the speech plans and speech output. Asynchronous events can also occur in normal listening conditions when speech is dysfluent. For example, stuttered speech may arise because PWS initiate speech before planning is complete (Howell, 2010). Planning can continue during the time that the speech is uttered. If an efferent copy of the plan was taken at the point at which speech was initiated, then this would be asynchronous with the eventual auditory output. Differencing the timing patterns of asynchronous inputs (DAF and stuttered speech) would not cancel. Speakers may use the signal that is selectively generated when inputs are asynchronous as an indication to reduce speaking rate. Consistent with this, DAF slows the speaking rate of fluent speakers, as first reported by Lee (1950). DAF has been used to slow the speaking rate of PWS (Goldiamond, 1965) as a fluency intervention that would allow time to complete speech-planning. The reduction in speaking rate would remove the condition that led to the fluency problem and inputs that were asynchronous would reinstate to synchrony. 
Two observations support the view that slowing speaking rate puts plans and outputs back into synchrony and allows fluency to be regained (Howell, 2010): 1) Empirical reports show that the reduced speaking rate under DAF improves fluency (Brendel, Lowit \& Howell, 2005; Goldiamond, 1965; Howell, Wingfield \& Johnson, 1988; Ryan, 1974). Whilst PWS can achieve fluency gains when they increase their overall speaking rate under AAF (Howell et al., 1987; Kalinowski, Armson, Stuart \& Graccco, 1993), speaking rate is low in the vicinity of segments that have a high likelihood of attracting stuttering (Howell \& Sackin, 2000). For instance, DAF always leads to stressed vowels being prolonged under different speaking rates and DAF delays (Kalveram, 2001; Kalveram \& Jäncke, 1989); 2) voluntary reductions in speaking rate in normal listening conditions enhance the fluency of PWS (Adams, Lewis \& Besozzi, 1973; Andrews, Howie, Dozsa \& Guitar, 1982; Janssen \& Wieneke, 1987; Perkins, Bell, Johnson \& Stochs, 1979; Vanryckeghem, Glessing, Brutten \& McAlindon, 1999).

The cerebellum has been proposed as the site of timing control structures that determine whether inputs during vocalization are synchronous, and if they are not, to initiate a slowing response. Howell and Sackin (2002) used Wing and Kristofferson's (1973) task to investigate how the cerebellum operates under DAF (i.e. when inputs are asynchronous). In the task, participants attempt to produce motor outputs isochronously (i.e. at equally timed intervals). Wing and Kristofferson (1973) decomposed the variance in the responses into motor (Mv) and timekeeper (clock variance, Cv). Ivry (1997) showed that patients these variance components are mediated by different parts of the cerebellum since lesions in the lateral or the medial parts of the cerebellum led to deficits in Cv or Mv respectively. Speakers in Howell and Sackin (2002) produced syllables isochronously under normal (synchronous) and DAF (asynchronous) feedback conditions. Only Cv increased under DAF compared to normal feedback. From this, Howell and Sackin (2002) proposed that the timekeeper in the lateral cerebellum detected when inputs were asynchronous. Watkins et al.'s (2008) fMRI study reported increased activity in the cerebellum under DAF, which is also consistent with the view that the lateral cerebellum identifies asynchronous inputs that could then be used to trigger a rate reduction that would improve the fluency of PWS ${ }^{1}$. 
VTF could not access the synchrony-detection mechanism since vibration is not transmitted via the cerebellum. Nevertheless, VTF improves the fluency of PWS (Kuniszyk-Jozkowiak et al., 1996; Snyder et al., 2009; Waddell et al., 2012). This suggests that rate control by the cerebellum is not the only way to improve fluency. Also consistent with a second fluency-inducing mechanism, AAF that is synchronous with speech, such as real-time frequency-shifted speech, FSF (Howell et al., 1987) has ameliorative effects on fluency. Furthermore, VTF should not affect speech timing if the lateral cerebellum controls rate. This second fluency-enhancing mechanism could reside in the post-thalamic structures that are available to VTF and synchronous AAF (i.e. FSF).

The VTF literature generally supports the view that the second fluency-enhancing mechanism affects speech control of PWS in a different way from that which arises when asynchronous auditory inputs activate cerebellar timing mechanisms. Synchronous VTF (Snyder et al., 2009; Waddell et al., 2012) enhances fluency. Neither of these VTF studies looked at speaking rate and they did not investigate asynchronous VTF. However, Kuniszyk-Jozkowiak et al.'s (1996) study: 1) demonstrated that DAF has more effect on speaking rate than does asynchronous VTF. It may be that only DAF invokes the lateral cerebellar mechanism and hence fluency is affected in a different way from when it arises from the second fluency-enhancing mechanism; 2) reported that speaking rate did not differ between synchronous VTF and synchronous auditory feedback conditions. This would be expected if both employ a fluency-enhancing mechanism other than the cerebellum. On the other hand, based on the hypothesis of a second, non-cerebellar fluency-inducing mechanism that is not affected when inputs are asynchronous, it is unclear why delayed

VTF reduced stuttering rate and speech timing more than synchronous VTF and why this difference was more marked as feedback delays were increased (Kuniszyk-Jozkowiak et al., 1996). The present study aimed to check the first result (the high number of conditions in the present study prevented examination of delay effects).

\subsection{Predictions about the fluency-enhancing effects of VTF and about ancillary parameters that are manipulated}


Two predictions about the second fluency-enhancing mechanism are tested below: 1) VTF should lead to fluency improvements relative to noVTF control conditions (Snyder et al., 2009; Waddell et al., 2012). Improvements should be equivalent regardless of whether VTF is synchronous or asynchronous with voice; 2) Making VTF asynchronous with speech by delaying it should not affect speaking rate relative to synchronous VTF. This is because the cerebellum, that triggers the change in speaking rate, is not included in the vibratory pathways. Conditions with no-feedback change (baseline controls), immediate VTF, and VTF delayed by $200 \mathrm{~ms}$ were employed to test the predictions about synchronous versus asynchronous VTF.

Two extra factors were manipulated under VTF stimulation to provide additional information about vibration pathways (stimulation sites) or to allow further comparison with studies about the fluency-enhancing effects of AAF and VTF (amplitude variation):

1. Stimulation sites were hand, sternum and forehead. The hand allowed comparison with previous studies (VTF was delivered to the middle and centre fingers in Kuniszyk-Jozkowiak et al., 1996 and Waddell et al., 2012 respectively). The sternum was used because it is an unobtrusive location for VTF fluency aids. Vibration is transmitted via the main sensory trigeminal pathway when applied to the forehead or via the medial lemniscal pathway for the other sites. Hence inclusion of the forehead site tests whether VTF on one or both pathways affects the speech of PWS.

2. Kuniszyk-Jozkowiak et al. (1996), Snyder et al. (2009) and Waddell et al. (2012) did not control amplitude of VTF (it varied with the amplitude of the primary speech signal). However, amplitude of stimulation affects speech under DAF (Howell \& Archer, 1984). Furthermore, Jones and Striemer (2007) argued that participants could focus better on the primary speech signal and reduce DAF's disruptive effects, when the delayed sound was at low, as opposed to high, amplitude. Therefore, amplitude needs exploring with respect to VTF. It was hypothesized that fluency should be enhanced more when amplitude of VTF is high (Howell \& Archer, 1984; Jones \& Striemer, 2007), unless response to amplitude variation is another property that distinguishes the two fluency-enhancing mechanisms. 


\section{Materials and methods}

\subsection{Participants}

Eleven right-handed adult PWS (six male and five female) participated. Age ranged from 24 to 63 years (median age $=43$, mean age $=43.72$, standard deviation $=10.78)$. The inclusion criterion was that they had to stutter currently. Stuttering Severity Index 4, SSI-4 (Riley, 2009) ${ }^{2}$ scores were between 5 and 23. The study was approved by the UCL Experimental Psychology ethics committee.

\subsection{Procedure and instrumentation}

Participants read different 300-syllable extracts from 'The Life of Pi' (Martel, 2002) at one practice, three control and 18 conditions where feedback was manipulated. The 22 passages varied between 'fairly easy' and 'easy' on the Flesch (1948) Reading Ease Scale (scores between 75 and 85 ). No VTF was presented during the practice and three control readings which were administered before, in the middle of, and at the conclusion of testing) ${ }^{3}$. Three independent variables were manipulated in the VTF conditions: 1) Tactile stimulator placement (finger, sternum and forehead); 2 ) Synchronicity of VTF (immediate or 200-ms delay); 3) Amplitude of VTF (low = 0.5-1.0 m/ $/ \mathrm{s}^{2}$, medium $=1.5-2.0 \mathrm{~m} / \mathrm{s}^{2}$ and high $=2.5-3.0 \mathrm{~m} / \mathrm{s}^{2}$ ). The choice of amplitude ranges was partly governed by the sensitivity range of the accelerometer used (vibration below $0.5 \mathrm{~m} / \mathrm{s}^{2}$ was not detectable). Participants performed all 18 altered VTF conditions (3 stimulator placement x 2 synchronicity x 3 amplitude conditions). A Latin square was used to assign passages to conditions.

The order of the three tactile placement conditions was established at random. Placement condition was blocked (e.g. the six finger, then the six forehead followed by the six sternum conditions). Blocking in this way avoided having to reposition the tactile stimulator. Finally, the order of synchronicity and amplitude conditions within each placement condition was randomized.

Figure 1 summarizes the equipment layout. To generate VTF, speech was amplified (pre-amp, M-Audio, Audiobuddy, amplifier, Quad, 520) and delivered to a 'Woojer' miniature wearable subwoofer, $(<\mathrm{http}: / /$ www.woojer.com $>)$. The Woojer had a resonant frequency of approximately 
$100 \mathrm{~Hz}$ which was audible in the high amplitude conditions. Consequently, the speech input was low-pass filtered (Barr \& Stroud, Variable Filter EF3) before transmission to the Woojer (cutoff $180 \mathrm{~Hz}$ and roll-off $48 \mathrm{~dB} /$ octave). The driving signal was input to a PC for calibration and monitoring using Audacity software (<http://www.audacity.sourceforge.net>).

Figures 1 and 2 about here

The Woojer was attached to parts of the body with elastic straps. The stimulator placements were: centre finger of the participant's dominant hand (Waddell et al., 2012); between the second and fifth articular depressions of the sternum; centre of the forehead. Delay was zero in the immediate feedback control conditions. Speech Monitor software (<http://www.speechmonitor.org $>$ ) was used to generate a VTF delay of $200 \mathrm{msec}$.

Figure 2 summarizes the amplitude calibration process. Amplitude was calibrated using a lightweight digital accelerometer (Bosch BMA180, $<$ http://www.bosch-sensortec.com>) positioned $1 \mathrm{~cm}$ from the tactile stimulator (attached to the participant's skin with electrical tape). The accelerometer transduced vibration from the surface of the body, passed it via a Teensy USB microcontroller (model 3.1, <http://www.pjrc.com>) to the PC which displayed the amplitude in real time (Processing, <https://processing.org>). The participant read a text and the VTF generated was amplified and then adjusted to the required intensity range $\left(0.5-1 \mathrm{~m} / \mathrm{s}^{2}, 1.5-2 \mathrm{~m} / \mathrm{s}^{2}\right.$ or $\left.2.5-3 \mathrm{~m} / \mathrm{s}^{2}\right)$ using a Shure, FP11 Automatic Gain Controller (AGC). 


\subsection{Statistical analysis}

The speech files in the control and experimental conditions were analysed using Speech Filing System, SFS (Huckvale, 2013). The following measurements were obtained in all conditions: 1) percentage of syllables stuttered (\%SS). Stuttering events were defined as blocks, part-word repetitions or prolongations (Riley, 2009). The number of stuttering events was divided by the number of syllables and converted to a percentage; 2) Riley's (2009) procedure was used to count syllables and these were then used to calculate speaking rate in syllables produced per second (SPPS); 3) the duration of each recording.

Ten percent of the recordings were re-estimated by the first two authors (the first author made the original assessments). For intra-rater reliability, the average intraclass correlation coefficient was 0.998 for $\% \mathrm{SS}(95 \%$ confidence intervals from $0.996-0.999, F(23,24)=611.855, p$ $=0.000)$ and 0.945 for SPPS (95\% confidence intervals from $0.873-0.976, F(23,24)=17.756, p=0.000)$. For inter-rater reliability, the average measure intraclass correlation coefficient was 0.997 for $\% \mathrm{SS}(95 \%$ confidence interval from $0.992-0.998, F(23,24)=287.918, p=$ $0.000)$ and 0.954 for SPPS 95\% confidence interval from $0.893-0.961(\mathrm{~F}(23,24)=21.598, p=0.000)$.

\section{Results}

\subsection{Stuttering rate $(\% \mathrm{SS})$}

The \%SS and SPPS data for individual participants are presented in Tables 1 and 2 for the control and experimental conditions respectively.

Table 3 shows individual participant rates of \%SS at baseline (i.e. pre-testing control condition) and during experimental conditions (an average of all experimental conditions was taken). 
The moments of the probability distribution of \%SS (mean, variance, skewness and kurtosis) are given in Tables 4 and for the control and experimental conditions respectively. Table 4 shows that, for $\%$ SS, the control data varied across participants but the amount of variation was about the same across these respective conditions.

Tables 4, 5 and 6 about here

Control conditions were compared first to establish whether receiving VTF in the intervening conditions affected \%SS. Wilcoxon Signedranks tests were performed as the data were not normally distributed (Shapiro-Wilk below 0.05). Pre/post, pre/mid and mid/post comparisons were made and Bonferroni correction was applied (adjusted $p$ value was 0.017). Table 6 shows that none of the differences were significant. In this analysis, there were no carry-over effects from the experimental to the control conditions.

The next analysis determined whether $\%$ SS changed in the experimental VTF conditions relative to control conditions. The ratios of the mean of the three $\%$ SS control conditions to the mean of the $18 \%$ SS experimental conditions were calculated for each participant. Ratios greater than 1 indicated that $\% \mathrm{SS}$ in control conditions were higher than those in experimental conditions (VTF reduced \%SS). The transformation: 1) reduced individual variability (i.e. participants who stuttered relatively little in the control conditions were likely to experience a small improvement in the 
experimental conditions and vice-versa for participants who stuttered a lot); and 2) normalized the data distribution (Shapiro-Wilks test statistic was $>0.05$, skewness and kurtosis values were in the $+/-1$ range, Hartley's FMax indicated variances were similar). The ratio of control mean/experimental mean was significantly greater than $1\left(t(10)=2.687, p=0.023\right.$ with $\eta^{2}=0.647$, a medium effect $)$. Hence VTF significantly reduced $\%$ SS rate using a statistic where the magnitude of the reduction was proportional to the initial baseline \%SS.

Next, a within-subjects Analysis of Covariance (ANCOVA) was conducted to determine whether particular placements (finger, sternum, forehead), amplitudes (low, medium, high), and variations in synchronicity (immediate, delayed) affected \%SS differentially. Pre-test \%SS was used as the covariate to limit the influences of severity variations across participants. Mauchly's test indicated that sphericity was violated for the experimental data. Therefore, the degrees of freedom for the main effects and interactions reported below used revised Greenhouse-Geisser estimates. The main effects and interactions that were significant in the initial analyses were not significant in follow-up analyses (see Table 7 for statistics).

\subsection{Syllables produced per second (SPPS)}

SPPS scores (Table 3) were compared across the three control conditions to establish whether VTF led to carry-over effects on speaking rate. Shapiro-Wilk test indicated normal distribution, Hartley's FMax showed approximately similar variances and data were ratio in nature. Hence, paired samples t-tests were used with Bonferroni correction applied (the adjusted $p$ value was 0.017). None of the differences were significant (statistics are summarized in Table 8).

Tables 7 and 8 about here 
Next, the average SPPS across the control conditions was compared with the average SPPS across the experimental conditions. Criteria for parametric analysis were met (i.e. Shapiro-Wilks test indicated normal distribution, Hartley's FMax indicated approximately similar variances, ratio data). A paired-samples t-test showed that there was no significant difference between control and experimental conditions $(t(10)=-2.138, p$ $=0.058$ ), which indicated that VTF did not affect SPPS. The overall SPSS means for the control and experimental conditions were 2.7998 (SD 0.7283 ) and 2.8976 (SD 0.6786) respectively.

The ratios of SPPS for all three control/all 18 experimental conditions were not distributed normally (Shapiro-Wilks test statistic had $p<0.05$ ). Therefore, the parametric test conducted on ratios using \%SS was not conducted.

As indicated previously, there was no effect of SPPS across control conditions, which suggests that speaking rates were stable. Hence, an ANOVA, rather than an ANCOVA, was conducted to determine any effects of placement, amplitude, and synchronicity on SPPS. Mauchly's test indicated that sphericity was violated for two interactions (placement and sychronicity; placement, amplitude, and synchronicity) and hence the degrees of freedom for these interactions used revised Greenhouse-Geisser estimates. All main effects and interactions were non-significant $(p$ values were between $0.318-0.954$, see Table 9 for statistics). Of particular note is the lack of an effect of synchronous versus delayed VTF on SPPS: Mean SPPS was virtually identical in the immediate $(\mathrm{M}=2.9010 \mathrm{SD}=0.6962)$ and delayed conditions $(\mathrm{M}=2.8942$, $\mathrm{SD}=0.6632)$. Thus. delaying VTF did not affect speaking rate.

Table 9 about here 


\section{Discussion}

\subsection{Summary and implications of findings for accounts of fluency enhancement}

VTF reduced stuttering rate. This confirmed hypothesis one and replicated and extended previous reports (Kuniszyk-Jozkowiak et al., 1996; Snyder et al., 2009; Waddell et al., 2012).

Speaking rate was not affected by VTF in several analyses and confirmed hypothesis two. There was a non-significant trend for syllables spoken per second to reduce (slower speaking rate) over the control, rather than the VTF conditions. The present findings about rate are not consistent with Kuniszyk-Jozkowiak et al. (1996), who found that delaying VTF slowed speech.

The third hypothesis examined concerned whether vibration transmitted via the main sensory trigeminal CN (activated by vibration applied to the forehead) and non-CN pathways (activated by vibration applied to the fingers and sternum) had different effects. \%SS did not vary when the transducer was positioned on different parts of the body. Thus, both vibration pathways were equally effective in influencing fluency in the follow-up tests. The two projection pathways merge above the thalamus and it is likely that the second fluency-enhancement mechanism lies around this location (accessible by both pathways) since stimulation that affects each pathway is equally effective, as discussed in section 1.3.

The fourth hypothesis explored whether VTF had similar effects to DAF. VTF had a greater effect upon $\% \mathrm{SS}$ at low $\left(0.5-1 \mathrm{~m} / \mathrm{s}^{2}\right)$ and high $\left(2.5-3 \mathrm{~m} / \mathrm{s}^{2}\right)$ amplitudes than at the middle amplitude setting $\left(1-2.5 \mathrm{~m} / \mathrm{s}^{2}\right)$. With DAF, the effects on fluency are greater when amplitude is increased (Howell \& Archer, 1984). The different patterns seen when VTF (curvilinear effect) and DAF (monotonic effect) amplitude are another difference 
between the two forms of altered feedback. Also, previous findings suggest that the cerebellum affects fluency when inputs are asynchronous (Howell \& Sackin, 2002). Anatomical considerations indicate that VTF does not involve the cerebellum, but fluency is enhanced nevertheless. These observations suggest that there is a second mechanism, other than that involving the cerebellum that influences fluency. The different amplitude responses may also reflect the fact that VTF and DAF affect separate fluency-enhancing mechanisms (cerebellar and supra-thalamic respectively). The second mechanism could be employed with synchronous feedback (auditory or otherwise) and VTF that cannot engage the cerebellar mechanism. The observed lack of rate effects on VTF would be expected if extra-cerebellar mechanisms are involved. As well as the present behavioral considerations, the anatomical observations about VTF pathways covered in the introduction also support involvement of a mechanism other than the cerebellum as an explanation of the fluency improvements.

\subsection{Effect size comparison with literature.}

There was an average $\%$ SS reduction of $21.82 \%$ under VTF (a medium effect size). The effect observed here was less than in previous studies, e.g. Waddell et al. (2012) reported a 71-80\% reduction. Nor is it comparable with reductions under DAF or FSF (Lincoln et al., 2006 report that FSF leads to a reduction in stuttering by $40-85 \%)$.

One possible explanation is that previous studies that have used tactile stimulators with resonant frequencies around 230-250 $\mathrm{Hz}$, have unwittingly exposed participants to some auditory feedback (in addition to the intended VTF). It may, therefore, be auditory feedback that is partly responsible for the reduction in \%SS in previous studies (also see section 4.4.2 where the possibility that the Woojer did not adequately stimulate the Pacinian corpuscles is discussed). Future studies, incorporating, for example, a masking condition could help to determine whether VTF affects stuttering purely by influencing the somatosensory cortex or whether some degree of cochlear-mediated auditory feedback also plays a role. 


\subsection{Implications for Development of VTF Fluency Aid}

A clinical trial would be required before VTF could be seriously considered as an alternative form of fluency aid. Below we explore some of the strengths of VTF compared to auditory fluency aids (i.e. those making use of AAF) and speculate as to how the results of this study may contribute to the development of a VTF fluency aid in the future.

The usefulness of VTF as compared to auditory fluency aids may depend on whether a user is concerned with maximal symptom reduction or a natural speaking rate. If \% SS is targeted and rate influences can be tolerated, then it may be advisable to use DAF procedures. If procedures are required that do not affect speaking rate, then VTF may be favoured AS it avoids some of the drawbacks associated with DAF, for example the slowed speech sounds unnatural (Martin, Haroldson \& Triden, 1984). FSF would achieve this goal too, but users may prefer something that can be worn discretely rather than an in-the-ear device. In addition, instrumental (e.g. use of an accelerometer signal to drive VTF) and procedural factors (using participants with severe stuttering) may result in an increased effect of VTF on fluency compared to that estimated here. These considerations merit evaluation of fluency aids that incorporate VTF. A portable VTF device could overcome several of the limitations in DAF/FSF devices that are currently available as follows:

1) VTF appears to function effectively at low amplitudes (i.e. $0.5-1 \mathrm{~m} / \mathrm{s}^{2}$ ): High amplitude of VTF is not necessary to achieve optimum fluency gains. A portable device may reduce \%SS whilst being minimally distracting for users. Furthermore, lower amplitudes reduce the potential for negative side effects (e.g. muscle fatigue) that can occur when exposed to high amplitude vibration for extended periods (Rollin Stott, 2006). If lower amplitudes are employed, users could wear the device for longer periods without fatigue or the need to recharge batteries. 
2) Reduced Auditory Interference: VTF avoids the occlusion of the ear that occurs when using DAF/FSF devices. Occlusion is likely to influence perception of sounds and has implications both for perception of environmental hazards and speech.

3) Endogenous Speech Specific: VTF, when driven by a piezo contact microphone or accelerometer placed on or near the larynx, only represents vibrations caused by the speaker's larynx, not extraneous speakers or environmental noises, as happens when condenser/dynamic microphones are used. This would allow devices to work in noisy environments and for altered feedback to be limited to those occasions when the PWS is speaking.

4) Hidden: A contact microphone or accelerometer can receive sufficient input from vocal fold vibration to generate VTF when worn on or below the larynx (low enough on the throat to be positioned under, for example, a shirt collar). The rest of the components required for such a device (e.g. wires, battery and a tactile stimulator) could likewise all be hidden under clothing, if required.

VTF appears to function as effectively when delivered to discrete locations (e.g. sternum) compared to visible ones (e.g. fingers). A vibrating device that is in contact with the sternum, hidden under one's clothes, therefore seems viable. This fits with anecdotal reports that tactile aids for those with hearing/visual impairments work when in contact with chest/abdomen (Roeser \& Bauer, 2004).

5) Cost-effective: The components of a vibrotactile device would cost little and no electronic assembly would be required if off-the-shelf devices were used.

This study did not examine subjective considerations for the use of a VTF Fluency Aid. Qualitative studies could explore the experience of individuals using VTF devices in real-world settings. These could establish whether users would tolerate wearing a skin-mounted VTF device, 
whether extended vibration feedback is tolerable, and how best to design a device that is user-friendly (e.g. easy to mount, secure once attached, and simple to operate).

\subsection{Limitations}

\subsubsection{Participants}

Caution must be exercised, given the small sample size (11), in applying these findings to the population as a whole. The small sample size may have led to a lack of statistical power, leading to type 2 errors in the follow-up tests of main effects and interactions within the experimental condition data. Replication with a larger sample would be advisable, e.g. 15 participants would have achieved statistical power of 0.8 (in line with Waddell et al.'s (2012) study).

There was marked variability in the effect of VTF on \%SS amongst participants as has also been reported in previous studies. Here, for example, one participant experienced a $60 \%$ reduction in stuttering frequency while another experienced a $39 \%$ increase in stuttering frequency. Age of participants varied markedly. However, all participants were adult (older than 18 years) and stuttering was, therefore, unlikely to resolve.

This would not have been the case if younger participants had been used. Similarly, severity was low. Clinicians suggest that milder stuttering is more difficult to treat than severe forms. Hence focusing on the milder end of the spectrum is justified. Heterogeneity in samples of PWS in studies is a strength of this work.

The effect of VTF on neurogenic stuttering speech was outside the scope of the study. This would, however, be a useful area to explore in the future. For example the question of whether delayed VTF perturb neurogenic PWS in a way similar/dissimilar to DAF may shed more light on whether these two types of feedback act on a different fluency-enhancing mechanism. 


\subsubsection{Procedural factors}

Synchronicity (i.e. VTF with a $200 \mathrm{~ms}$ delay vs. immediate) did not significantly affect levels of \%SS ( $p=0.134)$. This is at odds with KuniszykJozkowiak et al. (1996). One possible reason for this discrepancy relates to the type of tactile stimulation used here. Tactile stimulators with resonant frequencies of 230-250 Hz (e.g. VBW32 Skin transducer, http://www.tactileresearch.org) have been used in previous studies (Waddell et al., 2012; Kuniszyk-Jozkowiak et al., 1996). These frequencies correspond approximately with the most sensitive frequency for Pacinian corpuscles (Griffin, 1990; Siegel \& Sapru, 2006). However, the Woojer device used in this study has a resonant frequency of around $100 \mathrm{~Hz}$. Consequently, the VTF delivered in this study may not have sufficiently stimulated these mechanoreceptors, potentially reducing its efficacy.

VTF was driven by a microphone, rather than an accelerometer output. This could diminish the efficacy of VTF since Waddell et al., (2012) reported that using an accelerometer to capture laryngeal vibrations produced a significantly greater reduction in \%SS as compared to microphonedriven VTF (80\% vs. 71\%, respectively). This may be because microphone-driven VTF captures background noises whereas accelerometer-driven VTF does not.

\section{Conclusions}

The effects of VTF on the fluency of PWS were confirmed. Speaking rate was not differentially affected between synchronous and asynchronous VTF conditions. Varying the amplitude has different effects for VTF and AAF. Together these findings suggest that VTF affects a different fluencyenhancing mechanim to AAF in PWS which, behavioral and anatomical findings suggest, is in post-thalamic pathways. The implications for a model of feedback control in PWS and for development of prosthetic VTF devices for PWS were discussed. 


\section{Footnotes}

${ }^{1}$ Another account has been offered by Kalinowski and Saltuklaroglu (2006). They hypothesized that the speech feedback under FSF and DAF lead to PWS perceiving the secondary speech signal as an exogenous sound that can activate mirror neurons responsible for fluent language rapidly. Side effects would be avoided if fluency can be induced in PWS under AAF without speaking rate being affected. However, as reported earlier, speaking rate is affected in difficult sections even though overall speaking rate may be unaffected (Howell \& Sackin, 2000; Kalveram \& Jäncke, 1989). Also, Hickok (2009) has raised several critical observations concerning the role of mirror neurons in human actions.

${ }^{2}$ SSI-4 scores: of all $(\mathrm{N}=11)$ participants were: median score $=10$, standard deviation $=5.66$. The eight participants who had "very mild" severity scores had median score $=9$, standard deviation $=2.17$. The three participants with "mild" severity scores had median score $=19$, standard deviation $=2.16$.

${ }^{3}$ The mid-point baseline condition was introduced after the first two participants had been tested. The data from these two participants were retained and the midpoint control was treated as missing data in analyses. 


\section{References}

Adams, M. R., Lewis, J. I. \& Besozzi, T. E. (1973). The effect of reduced reading rate on stuttering frequency. Journal of Speech and Hearing Research, 16, 671-675.

Andrews, G., Howie, P. M., Dozsa, M., \& Guitar, B. E. (1982). Stuttering: Speech pattern characteristics under fluency-inducing conditions. Journal of Speech, Language, and Hearing Research, 25(2), 208-216.

Belyk, M., Kraft, S. J. \& Brown, S. (2014). Stuttering as a trait or state - An ALE meta-analysis of neuroimaging studies. European Journal of Neuroscience, 41, 275-284.

Brendel, B., Lowit, A. \& Howell, P. (2005). The effects of delayed and frequency shifted feedback on speakers with Parkinson's disease. Journal of Medical Speech and Language Pathology, 12, 131-138.

Brown, S., Ingham, R. J., Ingham, J. C., Laird, A. R. \& Fox, P. T. (2005). Stuttered and fluent speech production: An ALE meta-analysis of functional neuroimaging studies. Human Brain Mapping, 25, 105-117.

Budde, K. S., Barron, D. S. \& Fox, P. T. (2014). Stuttering, induced fluency, and natural fluency: A hierarchical series of activation likelihood estimation meta-analyses. Brain and Language, 139, 99-107.

Caetano, G. \& Jousmaki, V. (2006). Evidence of vibrotactile input to human auditory cortex. NeuroImage, 29, 15-28.

Cherry, C. \& Sayers, B. (1956). Experiments upon the total inhibition of stammering by external control, and some clinical results. Journal of Psychosomatic Research, 1, 233-246.

Edge, A. L., Marple-Horvat, D. E. \& Apps, R. (2003). Lateral cerebellum: Functional localization within crus I and correspondence to cortical zones. European Journal of Neuroscience, 18, 1468-1485. 
Flesch, R. F. (1948). A new readability yardstick. Journal of Applied Psychology, 32, 221-233.

Goldiamond, I. (1965). Stuttering and fluency as manipulatable operant response classes. In: L. Krasner, P. L. Ullmann, eds. Research in behavior modification. New York: Holt, Rinehart \& Winston, 106-156.

Griffin, J. W. (1990). Development and pathological processes in the peripheral nervous system. In: G. M. McKhann, ed. New Issues in Neuroscience, 2, 5-14.

Hendelman, W. J. (2005). Atlas of functional neuroanatomy. Boca Raton: CRC Press.

Hickok, G. (2009). Eight problems for the mirror neuron theory of action understanding in monkeys and humans. Journal of Cognitive Neuroscience, 21(7), 1229-1243.

Howell, P. (2004). Effects of delayed auditory feedback and frequency-shifted feedback on speech control and some potentials for future development of prosthetic aids for stammering. Stammering Research: An on-Line Journal Published by the British Stammering Association, $1(1), 31$.

Howell, P. (2010). Recovery from Stuttering. New York: Psychology Press.

Howell, P. \& Archer, A. (1984). Susceptibility to the effects of Delayed Auditory Feedback. Perception and Psychophysics, 34, $296-302$.

Howell, P. \& El-Yaniv, N. (1987). The effects of presenting a click in syllable-initial position on the speech of stutterers: Comparison with a metronome click. Journal of Fluency Disorders, 12, 249-256.

Howell, P., El-Yaniv, N. \& Powell, D. (1987). Factors affecting fluency in stutterers when speaking under altered auditory feedback. In: H. Peters \& W. Julstijn, eds. Speech Motor Dynamics in Stuttering. New York: Springer Press, 361-369. 
Howell, P. \& Lu, C. (2016). Facts and theories about stuttering. In: P. van Lieshout, B. Maassen, \& H. Terband, eds. Speech motor control in normal and disordered speech: Future developments in theory and methodology. Rockville, MD: ASHA Press, 147-172.

Howell, P., \& Sackin, S. (2000). Speech rate manipulation and its effects on fluency reversal in children who stutter. Journal of Developmental and Physical Disabilities, 12, 291-315.

Howell, P. \& Sackin, S. (2002). Timing interference to speech in altered listening conditions. Journal of the Acoustical Society of America, 111, 2842-2852.

Howell, P., Wingfield, T. \& Johnson, M. (1988). Characteristics of the speech of stutterers during normal and altered auditory feedback. In: W. A. Ainsworth \& J. N. Holmes, eds. Proceedings Speech 88, Vol. 3, Edinburgh: Institute of Acoustics, 1069-1076.

Huang, C. M., Liu, G. \& Huang, R. (1982). Projections from the cochlear nucleus to the cerebellum. Brain Research, $244,1-8$.

Huckvale, M. (2013). Speech Filing System. Available at <http://www.phon.ucl.ac.uk/resource/sfs/ $>$

Ivry, R. 1997. Cerebellar Timing Systems. In: J. Schmahmann, ed. The Cerebellum and Cognition. San Diego, CA: Academic Press, 555-573.

Janssen, P., \& Wieneke, G. (1987). The effects of fluency inducing conditions on the variability in the duration of laryngeal movements during stutterers' fluent speech. In Speech motor dynamics in stuttering (pp. 337-344). Springer.

Jones, J. A. \& Striemer, D., 2007. Speech disruption during Delayed Auditory Feedback with simultaneous visual feedback. Journal of the Acoustical Society of America, 122, 135-141.

Juliano, S. L. \& Mclaughlin, D. F. (1999). Somatic senses 2: Discriminative touch. In: Cohen, H. (ed). Neuroscience for Rehabilitation. $2^{\text {nd }}$ Ed. Philadelphia: Lippincott Williams \& Wilkins, pp. 111-130.

Kalinowski, J. S. \& Saltuklaroglu, T. (2006). Stuttering. San Diego: Plural publishing. 
Kalinowski, J., Armson, J., Stuart, A., \& Gracco, V. L. (1993). Effects of alterations in auditory feedback and speech rate on stuttering frequency. Language and Speech, 36(1), 1-16.

Kalveram, K. T. (2001). Neurobiology of speaking and stuttering. In Fluency Disorders: Theory, Research, Treatment and Self-help. Proceedings of the Third World Congress of Fluency Disorders (pp. 59-65).

Kalveram, K. T., \& Jäncke, L. (1989). Vowel duration and voice onset time for stressed and nonstressed syllables in stutterers under delayed auditory feedback condition. Folia Phoniatrica et Logopaedica, 4l(1), 30-42.

Kuniszyk-Jozkowiak, W., Smolka, E. \& Adamczyk, B. (1996). Effect of acoustical, visual and tactile echo on speech fluency of stutterers. Folia Phoniatrica et Logopaedica, 48, 193-200.

Lee, B. S. (1950). Effects of delayed speech feedback. The Journal of the Acoustical Society of America, 22, 824-826.

Lincoln, M., Packman, A., \& Onslow, M. (2006). Altered auditory feedback and the treatment of stuttering: A review. Journal of Fluency Disorders, 31, 71-89.

Martel, Y. (2002). Life of pi. Canongate Books.

Martin, R. R., Haroldson, S. K., \& Triden, K. A. (1984). Stuttering and speech naturalness. Journal of Speech and Hearing Disorders, 49(1), 5358. 
Niemer, W.T. \& Cheng, S. (1949). The ascending auditory system, a study of retrograde degeneration. Anatomy Research, $103,116$.

Perkins, W. H., Bell, J., Johnson, L., \& Stocks, J. (1979). Phone rate and the effective planning time hypothesis of stuttering. Journal of Speech and Hearing Research, 22, 747-755.

Riley, G. (2009). The Stuttering Severity Instrument for Adults and Children (SSI-4) (4th ed.). Austin, TX: PRO-ED.

Roeser, R. J. \& Bauer, P. (2004). Cochlear Implants. In: R. J. Roeser \& M. P. Downs, eds. Auditory Disorders in School Children: The Law, Identification, Remediation. New York: Thieme, 323-343.

Rollin Stott, J. R. (2006). Vibration. In: D. J. Rainford \& D. P. Gradwell, eds. Ernsting's Aviation Medicine. Boca Ranton: Taylor and Francis Group, 231-246.

Ryan, B. (1974). Programmed stuttering therapy for children and adults. Springfield: CC Thomas.

Siegel, A. \& Sapru, H. N. (2006). Essential Neuroscience. Baltimore: Lippincott, Williams \& Wilkins

Snyder G. J., Blanchet, P., Waddell, D. \& Ivy, L. J. (2009). Effects of digital vibrotactile speech feedback on overt stuttering frequency. Perceptual and Motor Skills, 108, 271-280.

Stuart, A., Xia, S., Jiang, Y., Jiang, T., Kalinowski, J., \& Rastatter, M. P. (2003). Self-contained in-the-ear device to deliver altered auditory feedback: Applications for stuttering. Annals of Biomedical Engineering, 31(2), 233-237. 
Vanryckeghem, M., Glessing, J. J., Brutten, G. J., \& McAlindon, P. (1999). The main and interactive effect of oral reading rate on the frequency of stuttering. American Journal of Speech-Language Pathology, 8(2), 164-170.

Waddell, D., Goggans, P. \& Snyder, G. (2012). Novel tactile feedback to reduce overt stuttering, NeuroReport, 23, 727-730.

Watkins, K. E., Smith, S. M., Davis, S. \& Howell, P. (2008). Structural and functional abnormalities of the motor system in developmental stuttering. Brain, 131, 50-59.

Wing, A. M. \& Kristofferson, A. B. (1973). Response delays and the timing of discrete motor responses. Perception \& Psychophysics, 14, 5-12.

Zhang, S., Sun, X. \& Jen, P.H.S. (1990). HRP study of neural projections of acoustic signal to the cerebellum of echolocating bats. Acta Theriologica.Sinica, 10, 110-113. 
Figure legends

Figure 1 Equipment layout for recording speech, generation of VTF and the amplitude calibration process.

Figure 2 Equipment layout for amplitude calibration process. 


\section{Significance}

This study identified some of the neural structures that can be stimulated to improve the fluency of people who stutter. The study advanced our understanding of these fundamental processes. Several features of vibrotactile commend its use in prosthetic devices. 


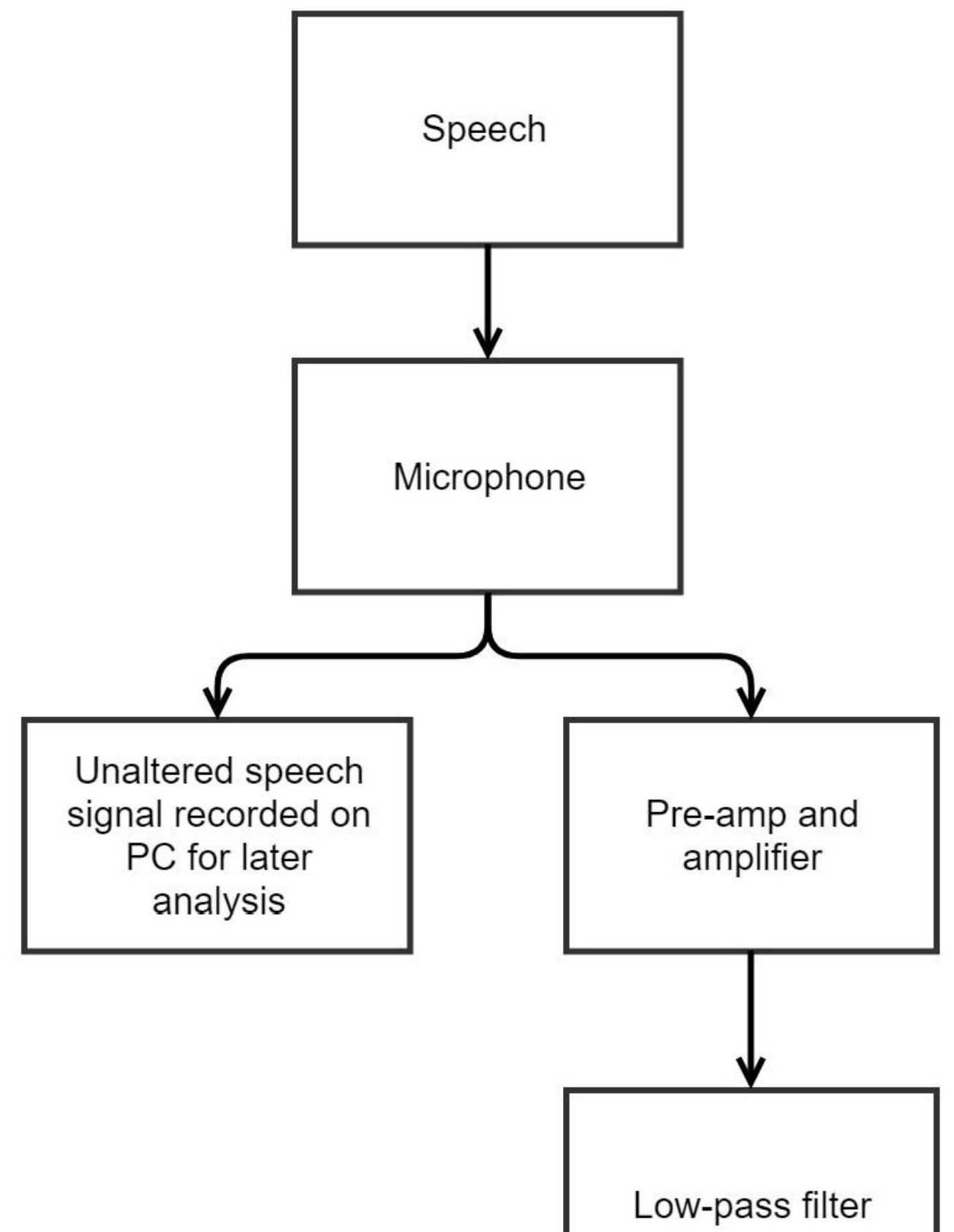




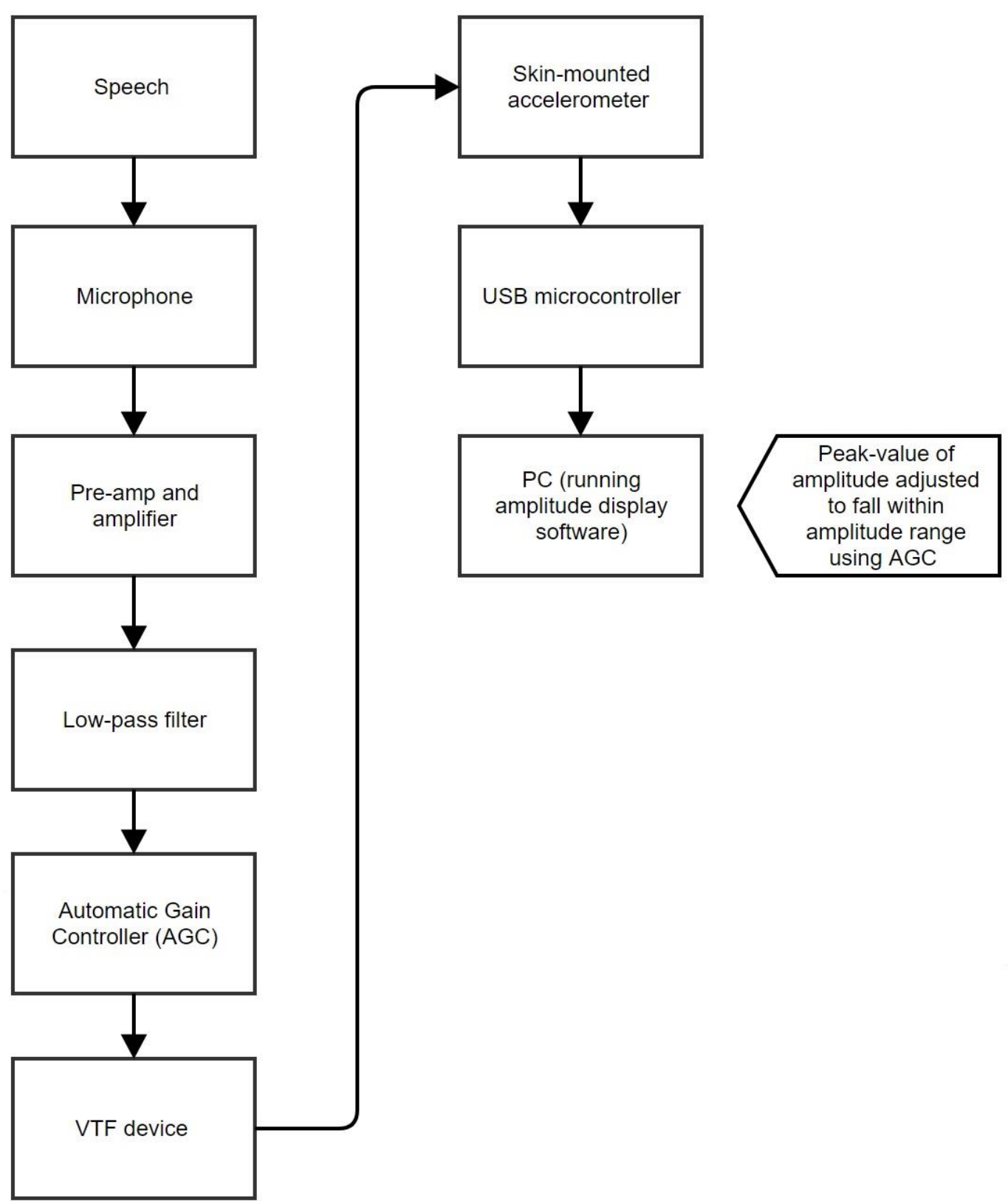




\section{Table 1}

Individual participant \%SS and SPPS data in control conditions (labelled in the left-most column).

\begin{tabular}{|c|c|c|c|c|c|c|c|c|c|c|c|}
\hline Control Condition & Participant & Participant & Participant & Participant & Participant & Participant & Participant & Participant & Participant & Participant & Participant \\
\hline & 1 & 2 & 3 & 4 & 5 & 6 & 7 & 8 & 9 & 10 & 11 \\
\hline \multicolumn{12}{|l|}{ Pre-testing Control: } \\
\hline$\% \mathrm{SS}$ & 7.800 & 33.100 & 4.230 & 1.890 & 20.333 & 2.251 & 4.459 & 1.303 & 1.736 & 0.315 & 1.603 \\
\hline SPPS & 2.305 & 1.013 & 3.384 & 2.365 & 2.065 & 2.853 & 1.614 & 3.118 & 3.488 & 2.516 & 4.394 \\
\hline \multicolumn{12}{|l|}{ Mid-testing Control: } \\
\hline$\% \mathrm{SS}$ & (not & (not & 1.260 & .000 & 22.013 & 1.320 & 0.676 & 3.175 & 1.623 & 0.310 & 0.342 \\
\hline SPPS & assessed) & assessed) & 3.131 & 2.395 & 1.894 & 3.613 & 2.374 & 3.166 & 3.305 & 3.376 & 3.852 \\
\hline \multicolumn{12}{|l|}{ Post-testing Control: } \\
\hline$\% \mathrm{SS}$ & 6.900 & 1.000 & 1.600 & 2.100 & 19.637 & 1.661 & 0.987 & 6.557 & 2.229 & 0.980 & 0.344 \\
\hline SPPS & 1.886 & 2.696 & 3.272 & 2.549 & 2.209 & 3.974 & 2.337 & 2.590 & 3.195 & 3.492 & 4.020 \\
\hline
\end{tabular}




\section{Table 2}

Individual participant \%SS and SPPS data in experimental conditions (labelled in the left-most column).

\begin{tabular}{|c|c|c|c|c|c|c|c|c|c|c|c|}
\hline Experimental & Participant & Participant & Participant & Participant & Participant & Participant & Participant & Participant & Participant & Participant & Participant \\
\hline Condition & 1 & 2 & 3 & 4 & 5 & 6 & 7 & 8 & 9 & 10 & 11 \\
\hline \multicolumn{12}{|l|}{ Finger Placement, } \\
\hline \multicolumn{12}{|l|}{ Low Amplitude, } \\
\hline \multicolumn{12}{|c|}{ Immediate Feedback: } \\
\hline$\% \mathrm{SS}$ & 9.500 & 26.400 & 1.870 & 2.230 & 16.190 & 0.000 & 1.299 & 0.987 & 1.639 & 0.322 & 0.000 \\
\hline SPPS & 2.041 & 1.128 & 3.364 & 2.686 & 2.371 & 4.080 & 2.429 & 3.060 & 3.470 & 2.919 & 4.186 \\
\hline \multicolumn{12}{|l|}{ Finger Placement, } \\
\hline \multicolumn{12}{|c|}{ Medium Amplitude, } \\
\hline \multicolumn{12}{|c|}{ Immediate Feedback: } \\
\hline$\% \mathrm{SS}$ & 8.300 & 33.500 & 1.870 & 2.230 & 18.361 & 1.316 & 0.633 & 3.883 & 1.014 & 0.645 & 0.331 \\
\hline SPPS & 2.176 & 0.808 & 3.451 & 2.700 & 2.156 & 3.848 & 2.391 & 2.776 & 3.507 & 3.261 & 4.049 \\
\hline
\end{tabular}


Finger Placement,

High Amplitude,

Immediate Feedback:

$\%$ SS

SPPS

2.612

11.000

1.600

4.980

15.484

1.954

0.64

3.236

0.318

0.317

0.322

$\begin{array}{lll}3.598 & 2.649 & 2.235\end{array}$

2.637

2.800

3.428

2.732

4.269

Finger Placement,

Low Amplitude,

Delayed Feedback:

$\% \mathrm{SS}$

SPPS

$$
5.400
$$

6.600

$$
2.230
$$

2.720

16.077
2.368

1.993
3.639

0.656
2.297

4.473
2.480

0.667
3.655

0.317

0.000

Finger Placement,

Medium Amplitude,

Delayed Feedback

$\% \mathrm{SS}$

$\begin{array}{llllllllllll}5.800 & 28.400 & 1.560 & 1.350 & 20.521 & 0.669 & 0.313 & 1.650 & 0.993 & 0.000 & 0.662 \\ 2.612 & 0.746 & 3.470 & 3.062 & 2.051 & 4.125 & 2.514 & 2.988 & 3.506 & 2.802 & 3.642\end{array}$


Finger Placement,

High Amplitude,

Delayed Feedback:

$\% \mathrm{SS}$

$7.600 \quad 11.800$

1.560

2.190

19.934

1.577

1.258

2.623

0.337

0.631

0.325

SPPS

2.589

1.509

$3.365 \quad 2.884$

2.096

3.798

2.243

2.745

$3.764 \quad 3.057$

3.992

Sternum Placement,

Low Amplitude,

Immediate Feedback:

$\% \mathrm{SS}$

6.30

SPPS

2.404

1.700
2.279

$\begin{array}{lll}3.100 & 0.710 & 23.127 \\ 2.940 & 2.641 & 1.988\end{array}$

4.000

0.000

2.640

1.613

$0.993 \quad 0.337$

Sternum Placement,

Medium Amplitude,

Immediate Feedback:

$\%$ SS

$\begin{array}{ll}10.800 & 3.300 \\ 2.896 & 2.267\end{array}$

SPPS

2.800
3.312

0.650
2.631

19.874

0.958

0.958

2.64

0.323

0.610

0.667

(1) 
Sternum Placement,

High Amplitude,

Immediate Feedback:

$\%$ SS

9.600

1.700

4.670

1.630

23.220

2.208

0.654

1.351

0.943

1.017

0.328

SPPS

2.195

2.455

$2.598 \quad 2.512$

$1.951 \quad 3.266$

1.75

3.258

3.566

3.239

4.147

Sternum Placement,

Low Amplitude,

Delayed Feedback:

$\% \mathrm{SS}$

SPPS

$8.200 \quad 1.600$
$2.503 \quad 2.833$

2.420

0.990

21.003

2.564

1.307

3.07

$3.104 \quad 2.628$

2.021

3.337

2.095

2.818

0.637
3.464

0.000

Sternum Placement,

Medium Amplitude,

Delayed Feedback:

$\% \mathrm{SS}$

$\begin{array}{lllllllllll}4.500 & 1.000 & 3.100 & 2.330 & 21.605 & 1.024 & 1.942 & 1.692 & 1.634 & 0.000 & 1.003 \\ 2.554 & 2.453 & 3.128 & 2.608 & 2.062 & 2.755 & 1.718 & 3.375 & 3.409 & 3.061 & 3.804\end{array}$

SPPS 
Sternum Placement,

High Amplitude,

Delayed Feedback:

$\% \mathrm{SS}$

5.600

3.100

3.400

1.350

23.867

1.294

0.631

3.268

0.000

$0.641 \quad 0.667$

SPPS

3.079

2.462

$2.635 \quad 2.575$

1.846

3.168

1.987

2.606

3.606

3.199

4.051

Forehead Placement,

Low Amplitude,

Immediate Feedback:

$\% \mathrm{SS}$

4.30

SPPS

2.944

7.300
2.597

1.680
2.681

0.960
2.736

17.722

1.399

0.621
2.348

0.974
3.566

0.623
3.358

1.37

3.222

0.332

Forehead Placement,

Medium Amplitude,

Immediate Feedback:

$\%$ SS

$\begin{array}{llllllllllll}4.900 & 5.000 & 2.600 & 2.940 & 21.835 & 1.987 & 0.322 & 2.589 & 0.651 & 0.000 & 0.000 \\ 2.530 & 1.899 & 3.045 & 2.458 & 2.002 & 3.844 & 2.376 & 3.187 & 3.542 & 3.514 & 4.201\end{array}$


Forehead Placement,

High Amplitude,

Immediate Feedback:

$\%$ SS

$10.500 \quad 1.610$

2.880

20.124

0.980

$0.321 \quad 2.007$

1.316

0.000

0.669

SPPS

2.828

1.705

3.693

2.554

2.222

3.970

2.34

$3.343 \quad 3.508$

3.190

4.063

Forehead Placement,

Low Amplitude,

Delayed Feedback:

$\%$ SS

SPP

$\begin{array}{lllllllllll}6.100 & 7.300 & 2.970 & 1.600 & 23.839 & 0.639 & 1.258 & 3.061 & 1.311 & 0.331 & 0.000 \\ 2.224 & 1.488 & 2.590 & 2.617 & 1.888 & 3.850 & 2.347 & 3.323 & 3.508 & 3.117 & 4.382\end{array}$

Forehead Placement,

Medium Amplitude,

Delayed Feedback:

$\% \mathrm{SS}$

$\begin{array}{llllllllll}12.800 & 1.960 & 0.990 & 21.362 & 0.980 & 0.318 & 2.922 & 1.423 & 0.000 & 0.313 \\ 1.667 & 3.261 & 2.442 & 1.998 & 3.865 & 2.579 & 3.294 & 3.378 & 3.240 & 4.357\end{array}$

SPPS

$1.839 \quad 1.667$

3.261


Forehead Placement,

High Amplitude,

Delayed Feedback:

$\begin{array}{llllllllllll}\% \mathrm{SS} & 4.000 & 2.600 & 2.270 & 0.670 & 19.683 & 0.993 & 1.270 & 2.251 & 0.311 & 0.333 & 1.010 \\ \text { SPPS } & 3.162 & 2.306 & 3.338 & 2.325 & 1.947 & 3.182 & 2.086 & 3.174 & 3.485 & 3.372 & 4.118\end{array}$


Table 3

Individual participant \%SS at in pre-testing control condition and average of all experimental conditions.

\begin{tabular}{|c|c|c|c|}
\hline Participant & Sex & Pre-testing Control $\% \mathrm{SS}$ & Experimental Conditions \%SS (average) \\
\hline 1 & Male & 7.800 & 6.583 \\
\hline 2 & Male & 33.100 & 9.756 \\
\hline 3 & Male & 4.230 & 2.404 \\
\hline 4 & Male & 1.890 & 1.856 \\
\hline 5 & Female & 20.333 & 20.213 \\
\hline 6 & Female & 2.251 & 1.474 \\
\hline 7 & Female & 4.459 & 0.800 \\
\hline 8 & Female & 1.303 & 2.515 \\
\hline 9 & Female & 1.736 & 0.875 \\
\hline 10 & Male & 0.315 & 0.453 \\
\hline 11 & Male & 1.603 & 0.387 \\
\hline
\end{tabular}


Table 4

Moments of the probability distribution (labelled in the columns) for the control \%SS and SPPS conditions (labelled in the rows).

\begin{tabular}{lcccc}
\hline Control Condition & Mean & Variance & Skewness & Kurtosis \\
\hline Pre-testing Control: & & & & \\
$\%$ SS & 4.235 & 38.222 & 2.742 & 7.822 \\
SPPS & 2.865 & 0.704 & 0.356 & 0.080 \\
& & & & \\
Mid-testing Control: & & & & \\
$\%$ SS & 3.413 & 49.553 & 2.898 & 8.530 \\
SPPS & 3.012 & 0.420 & -0.590 & -0.739 \\
& & & & \\
Post-testing Control: & & & & \\
$\%$ SS & 4.000 & 37.585 & 2.573 & 6.830 \\
SPPS & 3.071 & 0.467 & 0.195 & -1.524 \\
\hline
\end{tabular}




\section{Table 5}

Moments of the probability distribution (labelled in the columns) for the experimental \%SS and SPPS conditions (labelled in the rows).

\begin{tabular}{|c|c|c|c|c|}
\hline Experimental Condition & Mean & Variance & Skewness & Kurtosis \\
\hline \multicolumn{5}{|c|}{ Finger Placement, Low Amplitude, Immediate Feedback: } \\
\hline$\% \mathrm{SS}$ & 5.494 & 73.121 & 1.886 & 2.970 \\
\hline SPPS & 2.885 & 0.804 & -0.345 & 0.155 \\
\hline \multicolumn{5}{|c|}{ Finger Placement, Medium Amplitude, Immediate Feedback: } \\
\hline$\% \mathrm{SS}$ & 6.553 & 108.313 & 2.174 & 4.479 \\
\hline SPPS & 2.829 & 0.873 & -0.812 & 0.832 \\
\hline \multicolumn{5}{|c|}{ Finger Placement, High Amplitude, Immediate Feedback: } \\
\hline$\% \mathrm{SS}$ & 4.177 & 24.836 & 1.506 & 1.603 \\
\hline SPPS & 2.858 & 0.576 & 0.098 & 0.502 \\
\hline \multicolumn{5}{|c|}{ Finger Placement, Low Amplitude, Delayed Feedback: } \\
\hline$\% \mathrm{SS}$ & 3.739 & 21.471 & 2.145 & 5.288 \\
\hline SPPS & 2.963 & 0.387 & 0.233 & -1.425 \\
\hline
\end{tabular}


Finger Placement, Medium Amplitude, Delayed Feedback

$\% \mathrm{SS}$

SPPS

$\% \mathrm{SS}$

SPPS

SPPS

Sternum Placement, Medium Amplitude, Immediate Feedback:

$\% \mathrm{SS}$

4.047

2.373

SPPS

Sternum Placement, High Amplitude, Immediate Feedback:
Finger Placement, High Amplitude, Delayed Feedback:

Sternum Placement, Low Amplitude, Immediate Feedback:

92.173

0.836

1.695

$-1.143$

2.790

2.073

38.738

0.612

1.903

$-0.252$

3.205

$-0.700$

43.404

2.882

8.804

0.298

0.534

0.127
36.781

2.264

4.911

0.441

0.289

$-1.156$

46.426

2.574

6.868 
Sternum Placement, Low Amplitude, Delayed Feedback:

$\% \mathrm{SS}$

SPPS

Sternum Placement, Medium Amplitude, Delayed Feedback:

$\% \mathrm{SS}$

SPPS

Sternum Placement, High Amplitude, Delayed Feedback:

$\% \mathrm{SS}$

SPPS

Forehead Placement, Low Amplitude, Immediate Feedback:

$\%$ SS

SPPS

Forehead Placement, Medium Amplitude, Immediate Feedback: 
$\% \mathrm{SS}$

SPPS

Forehead Placement, High Amplitude, Immediate Feedback:

$\% \mathrm{SS}$

SPPS

Forehead Placement, Low Amplitude, Delayed Feedback:

$\% \mathrm{SS}$

SPP

Forehead Placement, Medium Amplitude, Delayed Feedback:

$\% \mathrm{SS}$

SPPS

Forehead Placement, High Amplitude, Delayed Feedback:
4.209

3.039

4.400

2.940

4.424

2.902

3.217

2.954
38.566

2.842

8.70

2.965

0.581

0.128

$-1.212$

4.606

$-0.994$

$0.588-0.290$

0.290

7.764

2.68

$-0.093$

$-0.919$

0.801

45.276

2.045

3.756

0.745

0.052

$-0.943$

$\% \mathrm{SS}$

31.079

3.081

9.830

SPPS

0.468

$-0.118$

$-0.907$ 
Table 6

Results of Wilcoxon tests for comparison of \%SS in the three control conditions. The control conditions being compared are indicated in column one.

\begin{tabular}{|c|c|c|c|c|}
\hline Conditions Contrasted & Median & Range & Z-score & $\mathrm{P}$-value \\
\hline Pre-testing Control \%SS & Pre Control $=1.890$ & Pre Control = 20.020 & -1.362 & 0.173 \\
\hline vs. & Mid Control = 1.260 & Mid Control = 19.300 & & \\
\hline \multicolumn{5}{|l|}{ Mid-testing Control \%SS } \\
\hline Pre-testing Control \%SS & Pre Control $=1.890$ & Pre Control $=20.020$ & -1.423 & 0.155 \\
\hline vs. & Post Control = 1.661 & Post Control $=19.300$ & & \\
\hline \multicolumn{5}{|l|}{ Post- testing Control \%SS } \\
\hline Mid-testing Control \%SS & Mid Control = 1.260 & Mid Control = 19.300 & -1.599 & 0.110 \\
\hline vs. & Post Control = 1.661 & Post Control $=19.300$ & & \\
\hline Post-testing Control \%SS & & & & \\
\hline
\end{tabular}


Table 7

Results of $3 \times 3 \times 2$ repeated measures ANCOVA for comparison of \%SS across VTF factors:

placement, amplitude, and synchronicity conditions. In all cases the pre-testing control \%SS

was used as a covariate.

Factor

F-ratio Degrees P-value Effect Size

of

freedom

Placement

$7.854 \quad 1.063,9.5 \quad 0.018$

0.466

69

Amplitude

25.32

$1.184,10$.

0.000

0.738

1

659

Synchronicity

2.715

1,9

0.134

ns

Placement\#Amplitude

28.9

$1.645,14$.

0.000

0.763 


\begin{tabular}{lcccc} 
Placement\#Synchronicity & 7.750 & $1.414,12$. & 0.010 & 0.463 \\
& & & & \\
& & & & \\
Amplitude\#Synchronicity & 9.334 & $1.327,11$. & 0.007 & 0.508 \\
& & & & \\
& & 945 & & \\
Placement\#Amplitude\#Sync & 17.81 & $1.477,13$. & 0.000 & 0.664 \\
& & & & \\
hronicity & 8 & 289 & \\
\hline
\end{tabular}




\section{Table 8}

Results of paired t-tests for comparison of SPSS in the three control conditions. The control conditions being compared are indicated in "column" one.

Conditions Contrasted

\begin{tabular}{|c|c|c|c|c|}
\hline & Median & Range & t-score & $\mathrm{P}$-value \\
\hline Pre-testing Control SPPS & Pre Control $=2.8530$ & Pre Control $=2.7795$ & -0.846 & 0.422 \\
\hline vs. & Mid Control = 3.1656 & Mid Control $=1.9580$ & & \\
\hline \multicolumn{5}{|l|}{ Mid-testing Control SPPS } \\
\hline Pre-testing Control SPPS & Pre Control $=2.8530$ & Pre Control $=2.7795$ & -1.270 & 0.233 \\
\hline vs. & Post Control $=3.1953$ & Post Control $=1.8112$ & & \\
\hline \multicolumn{5}{|l|}{ Post-testing Control SPPS } \\
\hline Mid-testing Control SPPS & Mid Control = 3.1656 & Mid Control $=1.9580$ & -0.634 & 0.544 \\
\hline vs. & Post Control $=3.1953$ & Post Control $=1.8112$ & & \\
\hline Post-testing Control SPPS & & & & \\
\hline
\end{tabular}


Table 9

Results of $3 \times 3 \times 2$ repeated measures ANOVA for comparison of SPPS across VTF factors: placement, amplitude, and synchronicity conditions.

Factor

ratio freedom

0.731

2,20

0.494

ns

Amplitude

Synchronicity

Placement\#Amplitude

Placement\#Synchronicity

Amplitude\#Synchronicity

Placement\#Amplitude\#Synchronicity

0.48

0.70

0.856

4,40

0.498

ns

1.162

$1.290,12.899$

0.318

ns

0.736

2,20

0.491

ns

$0.364 \quad 1.928,19.283$

0.692

ns 


\section{Highlights}

1. Differences between auditory and vibratory projection pathways were identified,

which were exploited to determine what differences occur when the pathways are

stimulated in people who stutter.

2. The fluency-enhancing effects of vibrotactile feedback were confirmed.

3. Differences in the slowing effects brought about by delaying vibrotactile and

auditory pathways were established.

4. Potential neural structures that may improve the fluency of people who stutter were

identified. 


\section{Significance}

This study identified some of the neural structures that can be stimulated to improve the fluency of people who stutter. The study advanced our understanding of these fundamental processes. Several features of vibrotactile commend its use in prosthetic devices. 